\title{
RFID Based Smart Lock implementation
}

\author{
Asst. Prof. Dr. Mohammed N. H. Al-Turfi \\ Computer Engineering Department, College Of Engineering, Al-Iraqia University, Iraq. \\ Email :mohammed-alturfi@yahoo.com \\ Engineer Rami Ahmed Adnan \\ Netwok Engineering Department, College Of Engineering, Al-Iraqia University, Iraq. \\ Email:ramiadnan01@gmail.com
}

Received on 11 January 2018 Accepted on 22 January 2018 Published on 14 May 2018

DOI: $10.30772 / q j e s . v 11 i 1.526$

\begin{abstract}
All people now a day are focusing on an important issue which is 'security' where all Rigid, worthy dealing with, and creditable companies pay a lot of attention for this problem. Therefore the cost, usage, portability must be taken in consideration. This work demonstrate the use of a smart, small, not expensive and the most important realistic portable lock with portable low consumption power supply and security coverage area with minimum side effect and maintenance.
\end{abstract}

This lock is not the representative for a key but it may represent a badge, an ID, or a credit card or social security card .... etc. So it's very close to the use of the human normal day life and hence it will not be a security burden since the security card may be embedded within other cards like car security card or credit card ...etc.

The system is implemented and tested with no need for a sophisticated material but easy equipment may stand enough to achieve the job with very accept able cost. The system achieves high security password and a master electronic key that cannot be copied within the available constraint and high control operating environment and a backup plan in case of key lost.

Keywords: Smart lock, Arduino, RFID.

\section{INTRODUCTION}

The RFID is a Capturing Data System that is used for Automatic Identification threw wireless channel, this is implemented using electromagnetic fields to identify accordingly tracked tags automatically and " which contain electronically stored (burned in) information "attached to objects is the general operating principle of RFID technology , and particularly in RFID locks [1].

The environment of the RFID contains normally two major parts, the Reader and the Tag, while there are two types of RFID Tags, Passive and Active, where passive one's collect the necessary energy transmitted from a nearby RFID reader's that generates the necessary operative radio waves hence the overall system coverage distance is small. While a local power source such a battery or solar cells must be available on board the active tags to power the system and hence the system may operate up to hundreds of meters from the RFID reader, unlike some other technologies where the tag need not to be within the line of sight of the reader, like the barcode, it may be embedded in the tracked object, RFID is replacing barcode technology and enjoys the major advantages of being line of sight problems independent and support objects scanning from distance.[2] 
Carrying and conveying data in suitable transponder media is the objective of any RFID system, generally tags task is to retrieve data, by classical, easy, or sophisticated readable means, with a time and place constrains to carry particular application and satisfy the needs. Therefore this technology is one of the most pushing up by both industrial and academic world where millions of advanced books; periodicals \& scientific magazines, CDs, DVDs, conferences and other electronic reading materials and articles are contained. It is a challenge to manage for librarians, managing such type and amount of huge collection. [3]

Many reasons for using RFID tags in many industries threw manufacturing and after sell, for example, an RFID tag attached to an equipment whether its heavy like a car or truck or light like a mobile phone or a home electric device during production can be used to track its progress through the assembly line or to provide mountainous and identification after sell. [1]

Size and mobility of RFID tags open new approach and made it available for attaching to cash, clothing, and possessions, or implanted in animals and people, the possibility of reading personally-linked information without consent has raised serious privacy concerns [2]. But on the other hand there are some sophistications available because RFID system is small and low cost and it can scan tags at rates of hundreds per second by the use of Radio Frequency (RF).[3]

These concerns resulted in standard specifications development addressing privacy and security issues. ISO/IEC 18000 and ISO/IEC 29167 use its own developed system for un traceability cryptography methods, tag and reader authentication, and over-the-air security and privacy. ISO/IEC 20248 specifies a data structure authentication which is digital signature for RFID and barcodes providing data, source and read method authenticity.[2]

With market stocks start from US\$6.96 billion in 2012 rises about $10 \%$ to reach US $\$ 7.77$ billion in 2013 , rises more than $15 \%$ in 2014 , the RFID market is worth US $\$ 8.89$ billion. This includes tags, readers, and software/services for RFID cards, labels, fobs, and all other form factors. The market value rises in 2015 to US $\$ 18.68$ billion and it reaches up to US $\$ 30$ billion in 2016 [4].

\section{System Constructions}

The system as shown in Fig(1) consists of two major parts, the S/W \& H/W one. The S/W where implemented and imbedded within the electronic card using $\mathrm{C}_{++}$programming for the following important reasons:

1-In-order to be able to modify or update the system software to handle new features and technologies.

$2-\mathrm{C}_{++}$script are one of the most powerful programming techniques that may stand to achieve high outputs and controlling abilities.

3-C++ programming may inter-prater with other programming languages and smoothly interface with them.

The H/W where implemented using three major devices:-

1-Arduino Electronic Card, which represents the heart of this system and the job controller with the following specifications:

- Operating Voltage(logic level): $5 \mathrm{~V}$

- Input Voltage (recommended): $6 \mathrm{~V}-12 \mathrm{~V}$

- Digital I/O Pins: 14 (of which 6 provide PWM output)

- Analog Input Pins: 8

- DC Current per I/O Pin: $40 \mathrm{~mA}$

- Flash Memory where it may be 16 KB (ATmega168) or 32 KB (ATmega328) where 2 KB used by boot-loader

- SRAM: 1 KB (ATmega168) or 2 KB (ATmega328)

- $\quad$ EEPROM: 512 bytes (ATmega168) or $1 \mathrm{~KB}$ (ATmega328)

- Clock Speed: $16 \mathrm{MHz}$ 

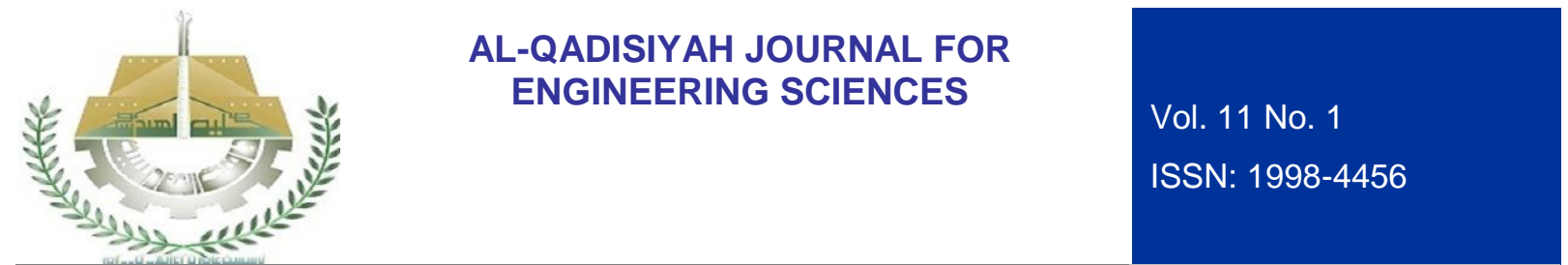

2-Relay :- which represents the job implementer and responsible for transducing the electric power into mechanical behavior threw control signal initiated by the Arduino triggered by the RFID card or the reader as shown in Fig(2).

3-RFID card reader :- Which is the job identifier where different types of RFID readers and cards with different operating frequencies may be used according to the implementation purposes and needs. But in general the RFID frequency ranges are all safe dealing with in \& out door since the operating power is relatively low "where passive RFID are zero power since the operation depends on the out collected power".

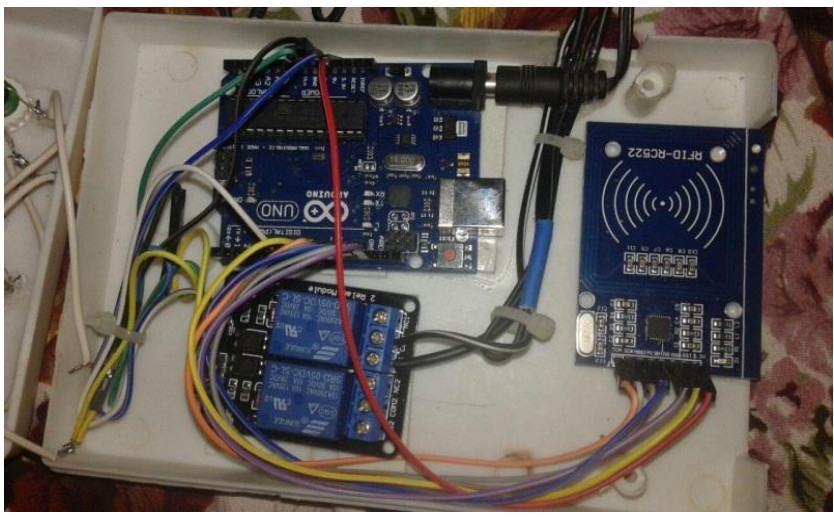

Figure 1: The system electronic board

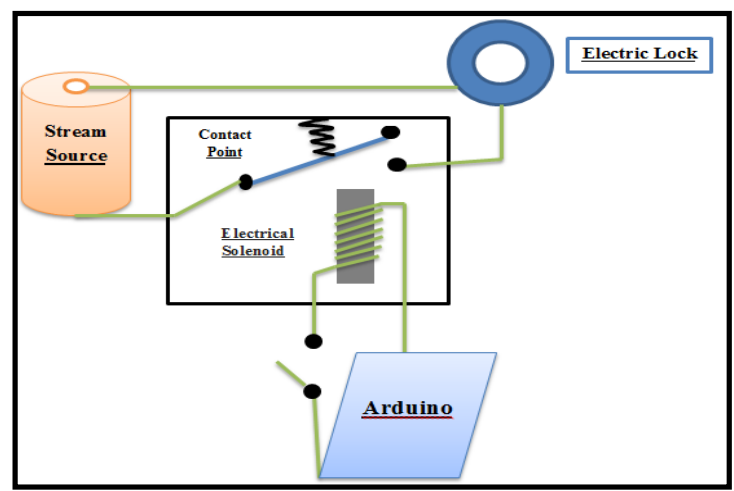

Figure 2:Relay operating principle and control

\section{System Implementation}

The system implementation must pass threw two phases Teaching (or Learning) \& Identifying where in the each phase some operations must be implemented in order not to miss the security issues and not to let any intrusion may hack the system.

\subsection{Learning Phase}

In this phase three operations must be taken in consideration and implemented in sequence to achieve the phase successfully:

1-There must be a specification for the code to be burned inside the RFID card which must be unique and representative for the person who carry the card and distinguish the level of authorization to specify card auxiliary functions .

2-There must be a code burner to down load the code to the card which has the ability to update it if necessary or duplicate it in case of losing or stealing with new tags and disable the old ones.

3-The number of users must be specified according to the reader memory size since each code is downloaded inside an RFID card must be duplicated and saved inside the reader data base for comparison and decision.

\subsection{Identification phase}

important constrain since the user can be identified if the user inside the system distance range elsewhere the system will not recognize the user RFID card.

The code identification process must check sections, person, and number, where incompatibility of any part means that the person is either try to access the wrong section-room or the person is not authorized to access this section-room. 


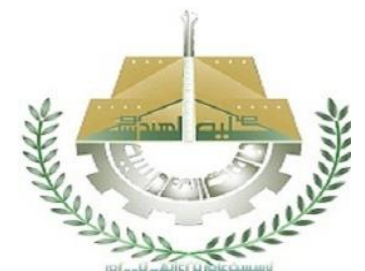

An alternative plan is adapted in case of losing or stealing the RFID card where the lock will not response for the right card unless a number will be entered after checking the card and hence the entrance will be secured.

\section{System Operation}

The operation starts when the RFID card passes within the distance range of the card reader, it takes the code inside the card by electro-magnetic induction since RFID communication tool that used to uniquely identify tagged object, possessions or people.

The code contains 16 digits each digit written in hexadecimal where the first 4 digits to specify the section, the second 4 digits specify the person level of authorization, while the 8 digits left specify the person number. In this case the number of probable cases is $16^{\wedge} 16$, this means that the correct number may be one of $1.8446734^{*} 10^{\wedge} 19$ choices, so the probability of breaking the lock is very low and hence the lock is relatively high secured.

When the system checks the user authentication depending on the card code and the secret number, the Arduino will trigger the relay to change status from OFF to ON where the power needed for the lock actuator will be reached and the system take the appropriate action.

The action still active until the system status changes where it backs to its previous state, hence the system original status may easily specified whether its active high (lock) or active low (unlock) depending on the case applications.

The system Pin Operation is the same as above where the RFID reader transmit the power through its reader antenna using Pin(11). When the specified RFID card is detected by the reader the reader will activate the system microcontroller using Pin $(10)$ where Pins $(9,10,11)$ are analog pins.

Relay has 3 inputs and 2 outputs. The system relay will be activated through Pin(7) where other two inputs are VCC which is 5 Volts and the Ground which is one of the Pins $(5,6,14)$.

Pins $(12,13)$ are Digital where they are inputs to the RFID reader, the supply voltage to the RFID reader is 3.3 Volts, Pin(9) is the RFID reader reset where this pin activate the system start over after each action to be taken, hence the reader overall number of Pins is seven. Finally $12 \mathrm{Volts}$ is the supply for the door lock which is the output from the relay to activate the actuator.

\section{CONCLUSIONS}

A Prototype home/ office automotive lock using RFID is designed and implemented with small size, low cost, high security, low power consumption, and the most important easy to maintain dedicated for automatic door control. This prototype operates in a safe frequency ranges that considered green specially the passive RFID where no power is available on board the card.

At the same time the system may be adapted to achieve multi tasks since the RFID tag may hold up to 4 Codes hence each code may hold certain task activated through certain relay activated by its own control signal to hold its task.

Side by side , and according to its small size it can be considered as a tariff card not only a key to lock since the RFID tag is embedded inside the car security remote control. Which means that this prototype can control various devices and developed for controlling lights and fans ...etc in smart green homes.

One more property is that the system may be programmed using other programming languages than $\mathrm{C}_{++}$, where a JAVA version of the system is implemented on the same board and equipments where the achieved task quality is identical to that programmed using $\mathrm{C}++$.

\section{REFERENCES}

1. Dr. Prabhat .P, Ms. K.D. Mahajan, "Application of RFID Technology in Libraries and Role of Librarian". 2013.

2. KamranA, Hanifa S, and Paul K, "RFID Applications: An Introductory and Exploratory Study", Faculty of Computing, Engineering \& Technology Staffordshire University Stafford, ST18 OAD, UK, 2013.

3. V. D. Hunt, A. Puglia and M. Puglia, "RFID-A Guide to Radio Frequency Identification", John Wiley \& Sons, Inc., Technology, 2012. 
4. Ann Cavoukian, Ph.D., "Privacy Implications of Radio Frequency Identification (RFID) Technology", Commissioner February 2004.

5. Zhi L, “Introduction to Microsoft Access 2003”, School of Information Fall/2006. 